\title{
Graft Tunnel Mismatch in Anterior Cruciate Ligament Reconstruction with Bone-Patellar Tendon-Bone Grafts
}

\author{
Joshua R Eskew, MD*, Daniel Miles, MD, Franky Davis, MD and Jeremy Bruce, MD \\ Department of Orthopaedic Surgery, University of Tennessee Health Science Center-Chattanooga, USA
}

\begin{abstract}
Anterior cruciate ligament (ACL) reconstruction with bone-patellar tendon-bone (BTB) autograft is a very common surgical procedure for patients with $\mathrm{ACL}$ deficient knees; however, itcan be a technically challenging operation with possible intra-operative complications. Graft-tunnel mismatch (GTMM) is a condition in which the ACL BTB autograft does not match the intra-articular and tunnel length. GTMM can result in compromised fixation for bone-to-bone healing when using a BTB autograft. The use of BTB autografts in a patient with patella baja or alt a can increase the likelihood of graft tunnel mismatch. Proper pre-operative planning can help avoid graft tunnel mismatch, however when facing mismatch there are techniques to salvage the graft and ensure bone-to-bone fixation.
\end{abstract}

\section{Keywords}

Anterior cruciate ligament, $\mathrm{ACL}$ reconstruction, Outcomes, Tendon-bone healing, Knee, Graft mismatch

\section{Introduction}

Anterior cruciate ligament $(A C L)$ rupture is a very common injury in active and athletic patients, and $A C L$ reconstruciton is the prefered surgical option for the ACL-deficient knee with funcitonal instability. While there are multiple options for reconstrcution grafts and fixation techniques, bone-patellar-tendon-bone (BTB) autologous grafts have long been considered the gold standard for graft options for the young and functioning patient [1]. ACL reconstruction with BTB autograft outcomes have shown superiority in positive quality of bone to bone fixation along with excellent clinical plus rehabilitation outcomes [1].

Dating back to 1963 , Dr. Robert Jones first used a BTB autograft during an $A C L$ reconstruction, and it has long been recognized as the preferred technique in $A C L$ reconstruction [2]. The novel technique showed increased postoperative strength, stiffness and bone to bone healing [2]. Using BTB autografts have been reported to have greater tensile strength compared to the native $A C L$, to have significatinly less anterior knee laxity and more stability compared to hamstring autografts [3-5]. BTB autograft revision rates have been reported to be significantly lower compared to hamstring autografts [6]. BTB autografts have also shown improved athletic function and a significant decrease risk in graft rupture at 2 years follow-up [7]. Aune, et al. [8] reported BTB autografts to have superior screw fixation compared to hamstring autografts, which allow for more optimal bone to bone healing and quicker return to rehabiliation. Krych, et al. [9] reported that BTB autografts experienced significantly less graft failure rates and a quicker return to play time. Autografts recoup
$80 \%$ strength, while allograts regain only $50 \%$ of strength [10]. Key studies have all concluded that undergiong ACL reconstruction with BTB autografts demonstated significantly lower rates of graft rupture, decreased levels of knee laxity, improved single-legged hop test results and increaed satisfaction postoperatively [10-13].

$A C L$ reconstruction with a BTB autograft does not come without risks [14]. Many orthopaedic surgeons will opt to not utilize BTB autografts due to the required advanced surgical technique and increased risk of anterior knee pain, patella fracture, patella baja, quadriceps shut down and long termrisk of osteoarthritis $[8,15,16]$. Conclusively, BTB autografts have also been shown to increase the likelihood of graft-tunnel-mismatch $[17,18]$.

Graft Tunnel Mismatch (GTMM) is a condition in which the $A C L$ graft length does not match the intra-articular and tibial tunnel length. This can be due to the ACL graft being too long (Figure $1 \mathrm{a}$ and Figure $1 \mathrm{~b}$ ) or too short (Figure 2). A

*Corresponding author: Joshua R Eskew, MD, Department of Orthopaedic Surgery, University of Tennessee Health Science Center- Chattanooga, Chattanooga, TN, USA

Accepted: May 23, 2020

Published online: May 25, 2020

Citation: Eskew JR, Miles D, Davis F, et al. (2020) Graft Tunnel Mismatch in Anterior Cruciate Ligament Reconstruction with Bone-Patellar Tendon-Bone Grafts. J Orthop Surg Tech 3(1):123-129 


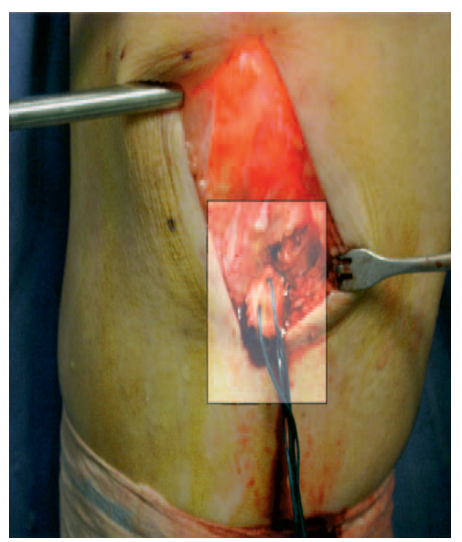

A

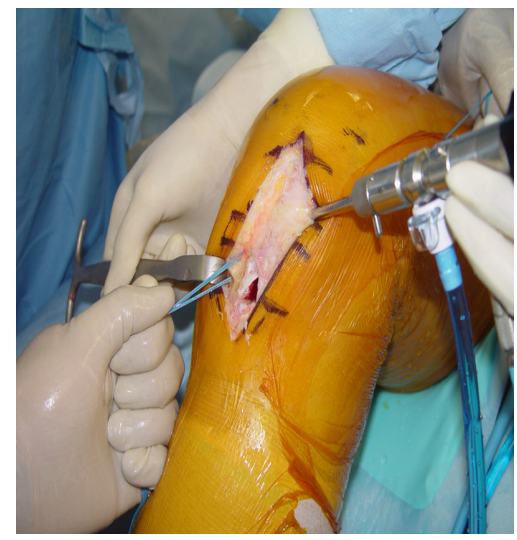

B

Figure 1(a,b): Example of ACL Graft that is too long.

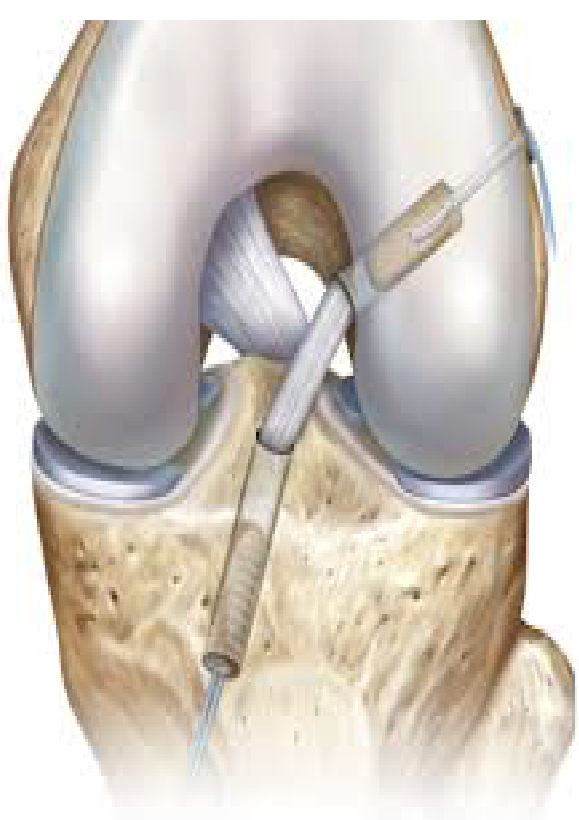

Figure 2: Example of ACL Graft that is too short.

graft that is too long is much more commonly encountered, especially when the graft is $>50 \mathrm{~mm}$ [19]. When GTMM is encountered there is a heightened risk of loss of bone to bone fixation with BTB autografts. Without optimal bone to bone fixation, optimal healing of the graft and bone will not occur. When the ACL graft is too long, it results in protrusion of the graft out of the tibial bone tunnel and necessitates removel of excess bone. This can result in compromised graft fixation and loss of optimal bone to bone healing. If the graft is too short in GTMM, screw fixation can push the graft up the tibial tunnel resulting in inadequate tension. Thus, it is important to check the length and tension of the graft prior to fixing it into place. Due to these increased risks, numerous bailout method options creating longer operative times, higher financial costs, and greater risks to each operative case- many orthopaedic surgeons will opt to use soft tissue grafts instead of BTB grafts.

\section{Incidence and prevalance}

$A C L$ injuries and $A C L$ reconstruction methods have become some of the foremost studied research topics in Orthopaedic Surgery and Sports Medicine due to the extensive number of injuries in athletes that require prompt reconstruction with quick recovery and rehabilitation. It has been estimated that over 100,000 ACL reconstructions are performed each year in the United Sates and with an annual incidence of 68.6 per 100,000 person years over the past twenty-one years [20]. The incidence of $A C L$ injuries ranged from 32 to 38 per 100,00 peope in Denmark, Germany and Sweden (countries with mandated $A C L$ reporting) [21]. The overall incidence of GTMM during ACL construction has been reportd to be $20 \%$ when utilizing BTB autografts and as high as $26 \%$ in BTB allografts causing a significant difficulty for surgeons intraoperatively $[18,19]$.

\section{Risk factors for graft tunnel mismatch}

Utilizing BTB autografts in ACL reconstruction does not come without unavoidalble risks. The most commonly reported negative outcomes from BTB autografts are anterior knee pain, sensation loss, patella fracture, patella baja, patella tendon rupture, loss of extension toruque, and graft tunnel mismatch. With methodical preoperative planning, BTB autograft complications can often be avoided or minimized. Risk factors for GTMM include but are not limited to: Previous knee joint trauma, degenerative tendon changes, patellar tendinopathy, patella alta, patella baja, Osgood-Schlatter disease, Sinding-Larson-Johansson syndrome, and patients who have obtained preoperative or intraoperative radiographic imaging without proper intra-articular length measurements. All patients undergoing $A C L$ reconstruciton should have preoperative radiography to assess all aspects of the knee joints including the location of the patella. Patella baja and alta have been reported to be risk factors that increase the likelihood of GTMM [22]. Using the 'Blumensaat's Method' via a lateral knee radiograph, the knee is flexed to 30 degrees. If the patella is superior to Blumensaat's line it is considered patella alta, and if it lies inferiorly, then it is considered patella baja. Patients with previous degenerative tendon changes and pa- 
Citation: Eskew JR, Miles D, Davis F, et al. (2020) Graft Tunnel Mismatch in Anterior Cruciate Ligament Reconstruction with Bone-Patellar Tendon-Bone Grafts. J Orthop Surg Tech 3(1):123-129

tellar tendinopathy should be worked up and be considered for an alternate graft source.

At presentation all patients should be initially examined with a thorough history and physical to identify risk factors that can could potentially lead to GTMM. Physicians should obtain detailed information of any prior joint trauma, surgery, infections, and degenerative changes. Patients that have a history suggestive of osteoarthritis or rheumatoid arthritis, should be worked up appropriately. Teenagers and young adults are more susceptible of having Osgood-Schlatter Disease and Sinding-Larson-Johansson syndrome. Patients with Osgood-Schlatter, also known as- tibial tubercle apophysitis will classically present in patients that repetitively run and jump with anterior knee pain over the anterior tibial tuberosity. Patients with Osgood-Schlatter Disease are prone to GTMM and ultimate graft failure due to false insertion of the graft into the tibia. Sinding-Larsen-Johansson syndrome is an overuse traction apophysitis of the inferior pole of the patella that is more common in growing active children, teenagers, and young adults. Patients should be questioned about inisidous onset of anterior knee pain during both rest and exacerbation when active. While the pain with Osgood-Schlatter disease was over the tibial tubercle, in patients with Sinding-Larsen-Johansson syndrome- the pain will be reside over the inferior patella. Patients with either suspected Osgood-Schlatter Disease or Sinding-Larsen-Johansson syndrome should undergo further assessment with radiography prior to $A C L$ reconstruction.

\section{Prevention}

\section{Radiographic/MRI assessment}

An initial radiographic assessment in patients with suspected $A C L$ rupture will undergo plain film and MRI radiography. Normal plain film radiographs should not preclude performing MRI on the affected knee. Upon confirmation of $A C L$ rupture, planning for operative $A C L$ reconstruciton should use several key measurements of the performed radiographs to minimize risk of GTMM.

Firstly, the patient's height should be carefully measured, as it has long been used to estimate the patient's ACL length. However, while the patient's height is significantly correlated to their ACL length, there is no significant association between patella ligament length and patient height [17]. This is critical, as Joyner, et al. [23] examined 138 total native ACL's and sought to quantify native $A C L$ length via Lateral X-ray Radiography. They found that the correlation between native $A C L$ length and Blumensaat's line length for males and females were both excellent ( 0.88 and 0.84 , respectively) [23]. They also found the correlation between native ACL length and patellar ligament length was very poor in both males and females (0.08 and 0.10 , respectively) [23]. They concluded that significant variability can be expected in $A C L$ and patellar ligament length and that lateral knee $X$-rays can serve as a simple and cost effective manner to estimate native ACL length [23]. Regardless of the type of graft being used, a patient's height should not be solely relied upon as the only estimate of native
$\mathrm{ACL}$ length to determine graft length, and lateral knee X-rays should be performed to help decrease GTMM risk by helping to better estimate native ACL length.

\section{Calculation of graft/tunnel length}

Using lateral X-ray films and MRI, the femoral tunnel (FT) length and $A C L$ length can be estimated. Once the femoral tunnel length and $A C L$ length are known, it is possibel to adjust the tibial tunnel (TT) length to match the overal graft length (OGL). However, it is important to remember that not all intra-articular ACL lengths are the same. It was originally thought that most, if not all, intra-articular $A C L$ lengths ranged between 25-30 millimeters, hoewever variability has been noted throughout literautre ranging from 21 to 42 millimeters $[19,24]$. While it would anatomically make sense that $\mathrm{ACL}$ length correlates with patella ligament length, Denti, et al. [25] demonstrated no statistically significant correlation between native $A C L$ and patellar ligament length.

Brown, et al. [26] examined 414 knee Magnetic Resonacne Images of knees with intact $A \mathrm{CL}^{\prime} \mathrm{s}$ and developed a linear regression formula to calculate the length of the ACL based on the patient's height:

\section{[ACL Length $=1.17 \times$ height (inches) -41.29$]$}

In the past, orthopedic surgeonsused the patient's height to calculate the paterllar legnth. patellar ligament length. However, the patella ligament length is not a reliable indicator for estimating $\mathrm{ACL}$ length. This is particularly concering if a surgeon is making a decision on estimated $A C L$ length based on patellar ligament due to multiple studies showing patellar length being close to 50.0 millimeters [27]. Especially when GTMM is significantly more common when the tendinous portion of the graft exceeds 50 millimeters [27]. Denti, et al. [25] showed that the average patella length was 45.5 millimeters +/- 4.7 millimetes with $25 \%$ being longer than 48 millimeters, while Shaffer, et al. [19] reported a mean patella ligament length of 48.4 millimeters +/- 6.0 millimetes with $20 \%$ being longer than 52.0 millimeters. Joyner, et al. [23] found an average patella ligament length of 49.2 millimetes $+/-7.0$ millimetes in males and an average of 44.4 millimeters $+/-6.0$ millimeters in females.

Other stuides have attempted to determine formulas to calculate tibial tunnel length and guide angle to accommodate the BTB autograft length. Kenna, et al. [28] proposed the " -50 " rule, estimating that the tibial tunnel length equals the overal graft length - 50.0 millimeters. Verma, et al. [27] proposed the " $\mathrm{N}+10$ " Rule, in which the length of the graft in millimeters plus 10 degrees is the optimal angle of the tibial guide (i.e. $50 \mathrm{~mm}$ graft $=60$ degrees). Miller, et al. [22] later proposed the " $\mathrm{N}+7$ " Rule, in which the length of the tendinous graft in millimeters plus 7 degrees is the optimal angle of the tibial guide (i.e. $50 \mathrm{~mm}$ graft $=57$ degrees). Finally, Olszewski, et al. [29] proposed the " $N+7 / N+2$ " rule, in which the tibula tunnel length is the patella ligment length plus 2 millimeters. While all of these stuedies are critically beneficial, they did not calculate the actual intra-articular length. Alas, Joyner, et al. [24] proposed a formula to calculate the Intra-Articular Graft Length: 


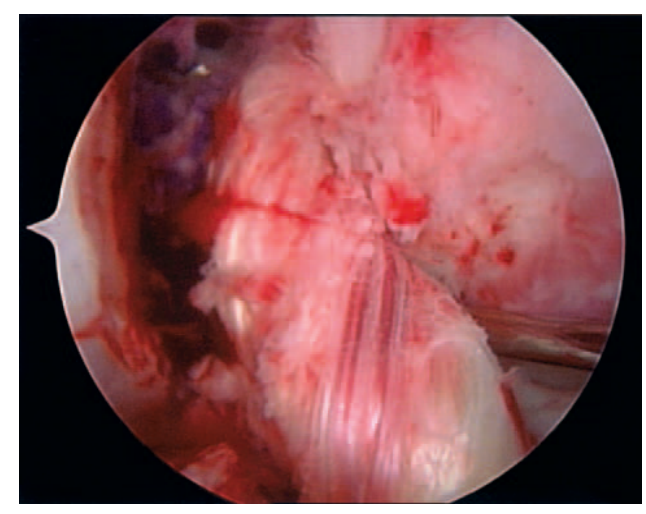

Figure 3: Example of bailout option: Twisting the $A C L$ graft.

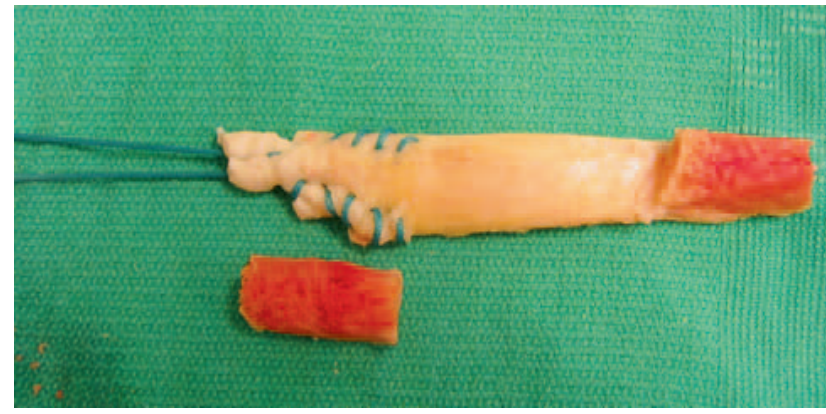

Figure 4: Example of bailout option: Free bone block.

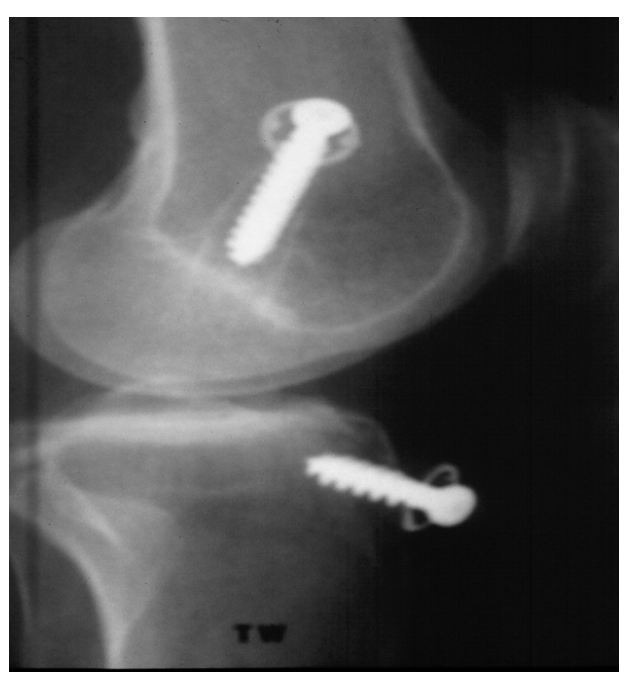

Figure 5: Example of bailout option: Tibial trough with screw and post.

[Graft Interligamental Length = Original Graft Length (Length Between Femoral Bone Plug + Tibial Bone Plug)]

\section{Graft Tunnel Mismatch Surgical Options}

Preoperative consideration should examine all risks for BTB complications including: age of the patient and age of the graft donor. Even with thorough preoperative planning and preparation to minimize the risk of GTMM during $A C L$ reconstruction, GTMM can still occur. When running into the complication of GTMM it is crucial to have bailout options.
There are numerous techniques that have been proposed to salvage the graft including; 1 ) Shortening Femoral Bone Plug Length; 2) Drilling Deeper Into the Femoral Tunnel; 3) Flipping the Graft; 4) Twisting the Graft and 5) Performing a Free Bone Plug.

\section{Twist the graft}

Verma, et al. [18] proposes twisting the graft if less than 12 $\mathrm{mm}$ or to free the bone if block is greater than or equal to 12 millimeters. Twisting the graft involves rotation 540 degrees to twist and shorten the tendinous portion of the graft which can shorten the graft on average by 5.4 millimeters (Figure 3 ).

\section{Flip the graft}

Barber, et al. [30] describe flipping the bone graft, which can theoreticlaly shorten the graft by 2-3 centimeters depending on the length of the bone plug.

\section{Free bone block}

For greater than or equal to 12 millimetes, Verma, et al. [27] also proposed a free bone block technique by stripping the bone block on the tibial tunnel portion and laying it over the soft tissue portion with an interference screw. Free bone block has been found to have superior biomechanical testing in the face of GTMM when compared to to the screw and post method [14] (Figure 4).

\section{Tibial trough with screw and post}

Tibial trough is another bailout option, in which you make a trough for a tibial bone plug and fix with a screw and post (Figure 5).

\section{Other options}

To shorten the femoral bone length will shorten the overall graft length, while also increasing the risk of compromising bone to bone fixation and healing. Drilling deeper into the femoral tunnel is simple but risks blowing out the outer cortex and can potentially cause graft impingement. Fowler, et al. [31] propose the free bone plug by placing an autograft cancellous core of bone into the tibial tunnel to improve tibial fixation.

\section{Case Examples Using Intra-Articular Graft Calculation}

\section{Patient \#1}

16-year-old Junior High School Football Player, Height: 5 feet, 10 inches, Father's Height: 5 feet, 9 inches diagnosed with ACL tear, Medial Meniscus Tear, Lateral Mensiscus Tear.

a. $\mathrm{OGL}=\mathrm{TT}+\mathrm{ACL}+\mathrm{FT}$

b. $O G L=T T+33 \mathrm{~mm}+25 \mathrm{~mm}$.

C. Estimation of TT to be $37 \mathrm{~mm}$, thus OGL should be $95 \mathrm{~mm}$.

d. MRI Patella Ligament $=52 \mathrm{~mm}$ (Bone Plugs $=20$ and 23 $\mathrm{mm})$.

e. $52 \mathrm{~mm}+20 \mathrm{~mm}+23 \mathrm{~mm}=95 \mathrm{~mm}$ for Original Graft Length. 
f. No GTMM during operation (Figure 6).

\section{Patient \#2}

Graft $=93 \mathrm{~mm}(23 \mathrm{~mm}+20 \mathrm{~mm}$ bone plugs and MRI Patella Length of $50 \mathrm{~mm}$ ).

a. $\mathrm{FT}=25 \mathrm{~mm}, \mathrm{ACL}$ templated to $33 \mathrm{~cm}$.

b. $\mathrm{OGL}=\mathrm{FT}+\mathrm{ACL}+\mathrm{TT} \rightarrow 93=25+33+\mathrm{TT}$

C. TT needs to be $35 \mathrm{~mm}$.

d. No GTMM during operation.

\section{Patient \#3}

17-year-old male Senior High School Football Player.

a. Height = 5 feet, 11 inches, $A C L$ and MCL tear.

b. Patella Ligament Length $=42 \mathrm{~mm}$ on lateral X-ray.

C. BTB graft only $75 \mathrm{~mm}$ with $20 \mathrm{~mm}$ plugs.

d. PL length ended up being only $35 \mathrm{~mm}$.

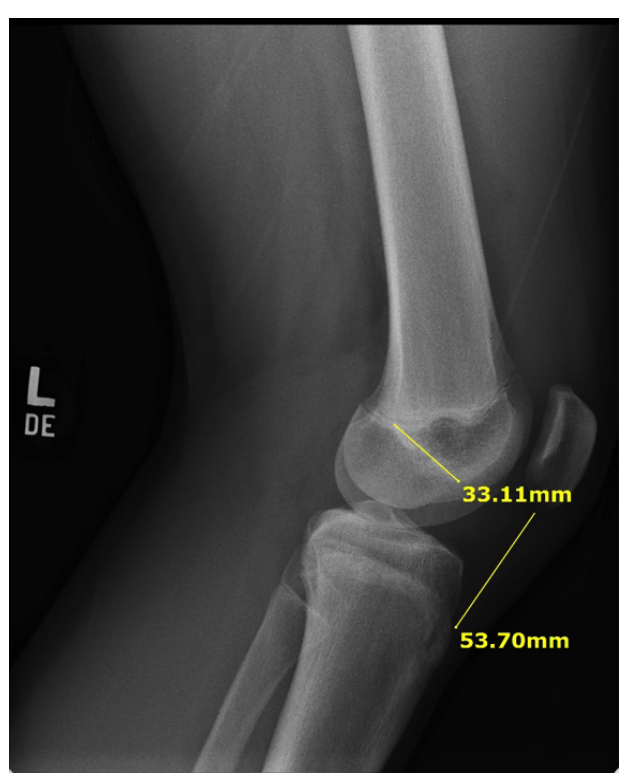

Figure 6: XR of Patient \#1.
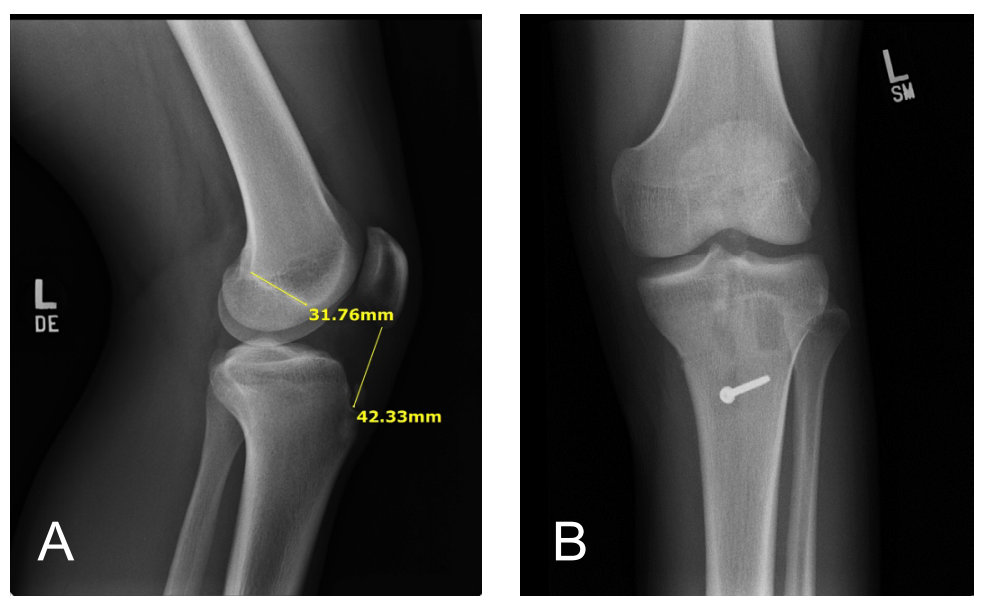

Figure 7: a) XR of Patient \#2; b) XR of Patient \#3. e. Attempt to make shorter femoral/tibial tunnel but graft still $10 \mathrm{~mm}$ short from outer cortex.

f. Trough in tibial tunnel was made to ensure screw captures and not pushing bone plug up tunnel with careful tapping of tibia because of blind tap.

g. Backed up with screw and soft tissue washer.

h. $\mathrm{MCL}$ also repaired with suture anchors (Figure 7a and Figure $7 b)$.

\section{Patient \#4}

18-year-old Senior High School Football Football Player.

a. Height: 5 feet, 7 inches.

b. Patella Tendon Length: $64 \mathrm{~mm}$ on lateral X-ray.

c. BTB graft $105 \mathrm{~mm}$ used intraoperatively.

d. Graft was too long leading to GTMM.

e. Longer tibial tunnel from 20 to $35 \mathrm{~mm}$ created but still had full bone graft protrusion, then shortened femoral bone plug from $24 \mathrm{~mm}$ to $18 \mathrm{~mm}$ with risk of femoral fixation.

f. Other possibility: Flipping graft (Figure 8).

\section{Patient \#5}

17-year-old athlete; Height: 5 feet, 7 inches with PL of $65 \mathrm{~mm}$ vs. Patient \#6: 18-year-old athlete; Height: 5 feet, 11 inches with PL of $35 \mathrm{~mm}$.

a. Important to remember that height is not a reliable indicator of ACL or Patella Ligament Length (Figure 9a and Figure 9b).

\section{Conclusion}

Anterior Cruciate Ligament reconstruction is a difficult and complex orthopaedic surigcal procedure for patients with $\mathrm{ACL}$ deficient knees. It is critical that all measures are taken prior to operation are performed to minimize risk and optimize success for each patient's outcomes. Graft Tunnel Mismatch is an intraoperative risk of graft failure that can be minimized by taking precautionary measures by properly es- 
timating graft legnth preoperatively. This can be done by assuming Blumensaat's Length $=\mathrm{ACL}$ Length with confirmatory measurement of both $\mathrm{ACL}$ length and patella ligament length via a preoperative lateral knee X-ray. The formulas above can then be used to approximate appropriate graft length and to minimize risk of graft tunnel mismathch. Madhu, et al. [32] agree that the dimensions of the graft and intra-articular length should be measured and assessed preoperatively. It is especially important to always remember that each patient's anatomy is unique. Patients with significant risk factors for graft failure should be appropriately examined thoroughly by history, physical and radiographic assessment for optimal graft choice and operative technique. All patients should be examined for significant patella baja or alta preoperatively with special consideration in those patients to be able to adjust the tibial tunnel accordingly.

When running into GTMM during surgery, we recommend that one does not immediately bail to an allograft. There are numerous options listed above as bailout methods to use with an autologous graft to give the patient the best oppor-

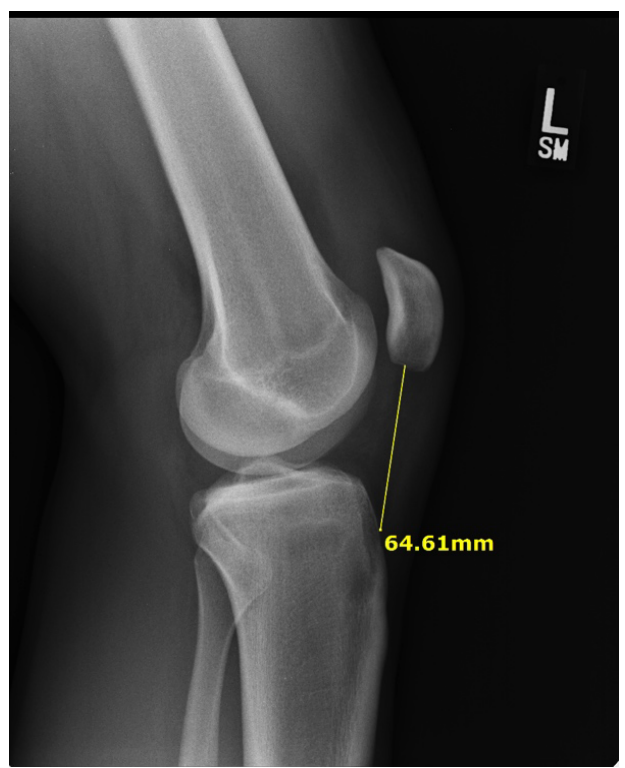

Figure 8: XR of Patient \#4. tunity for bone to bone healing. When using BTB autografts, it is of the upmost importance to speak and educate these patients about potential complications before they ever happen. Bone-patellar-tendon-bone autologous (BTB) grafts are not for everyone, and we recommend they be used in knee abusers, high level athletes and those with a closed physis. The hamstring autologous grafts (HS) are preferred in patients under 30-years-old with, those with an open physis, or active/ competitive patients over 30-years-old. Hamstring allografts are recommended for patients that are over 30-years-old, obese, as they have the fastest recovery back to normal daily activities.

\section{References}

1. Rue J, Lewis P, Parameswaran A, et al. (2008) Single-bundle anterior cruciate ligament reconstruction: Technique overview and comprehensive review of results. J Bone Joint Surg Am 90: 67-74.

2. Noyes FR, Butler DL, Grood ES, et al. (1984) Biomechanical analysis of human ligament grafts used in knee-ligament repairs and reconstructions. J Bone Joint Surg 66: 344-352.

3. Freedman KB, D Amato MJ, Nedeff DD, et al. (2003) Arthroscopic anterior cruciate ligament reconstruction: A metaanalysis comparing patellar tendon and hamstring tendon autografts. Am J Sports Med 31: 2-11.

4. Li S, Chen Y, Lin Z, et al. (2012) A systematic review of randomized controlled clinical trials comparing hamstring autografts versus bone-patellar tendon-bone autografts for the reconstruction of the anterior cruciate ligament. Arch Orthop Trauma Surg 132: 1287-1297.

5. Almekinders LC, Moore T, Freedman D, et al. (1995) Post-operative problems following anterior cruciate ligament reconstruction. Knee Surg Sports Traumatol Arthrosc 3: 78-82.

6. Lazarides AL, Alentorn-Geli E, Vinson EN, et al. (2018) Advanced patellar tendinopathy is associated with increased rates of bone-patellar tendon-bone autograft failure at early follow-up after anterior cruciate ligament reconstruction. Orthop J Sports Med 6: 2325967118807710.

7. MARS Group, MARS Group (2014) Effect of graft choice on the outcome of revision anterior cruciate ligament reconstruction in the Multicenter ACL Revision Study (MARS) Cohort. Am J Sports Med 42: 2301-2310.
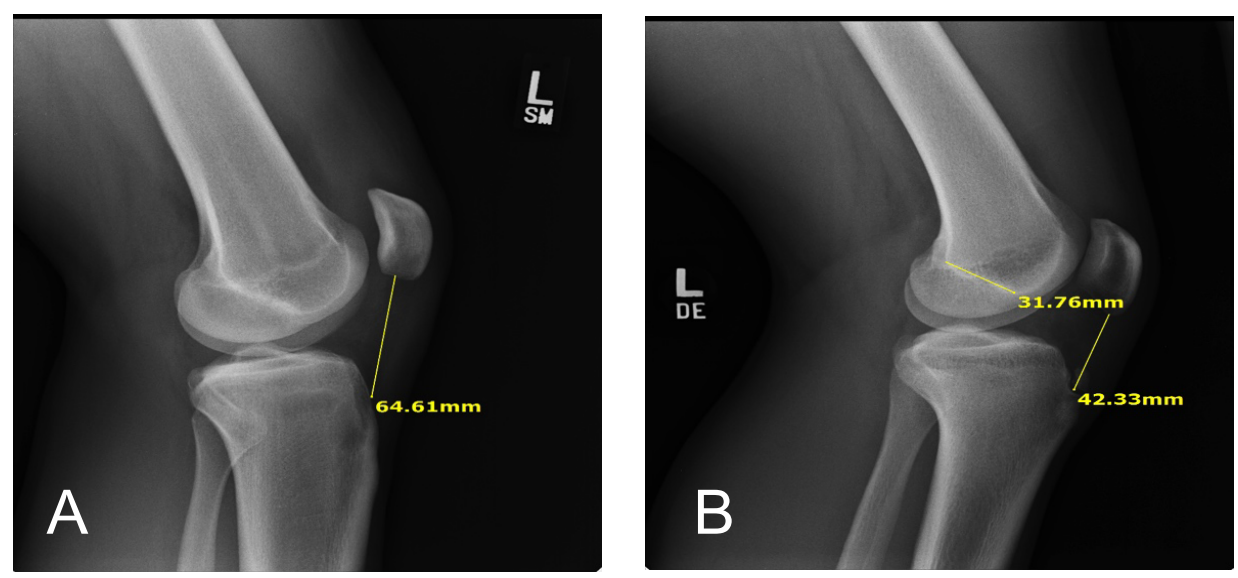

Figure 9: a) XR of Patient \#5; b) XR of Patient \#6. 
Citation: Eskew JR, Miles D, Davis F, et al. (2020) Graft Tunnel Mismatch in Anterior Cruciate Ligament Reconstruction with Bone-Patellar Tendon-Bone Grafts. J Orthop Surg Tech 3(1):123-129

8. Aune AK, Ekeland A, Cawley PW (1998) Interference screw fixation of hamstring vs patellar tendon grafts for anterior cruciate ligament reconstruction. Knee Surg Sports Traumatol Arthrosc 6: 99-102.

9. Krych AJ, Jackson JD, Hoskin TL, et al. (2008) A Meta-analysis of Patellar Tendon Autograft Versus Patellar Tendon Allograft in Anterior Cruciate Ligament Reconstruction. Arthroscopy 24: 292-298.

10. Bottoni CR, Smith EL, Shaha J, et al. (2015) Autograft versus allograft anterior cruciate ligament reconstruction: A prospective, randomized clinical study with a minimum 10-year follow-up. Am J Sports Med 43: 2501-2509.

11. Kraeutler MJ, Bravman JT, McCarty EC (2013) Bone-patellar tendon-bone autograft versus allograft in outcomes of anterior cruciate ligament reconstruction: a meta-analysis of 5182 patients. Am J Sports Med 41: 2439-2448.

12. Kaeding CC, Aros B, Pedroza A, et al. (2011) Allograft versus autograft anterior cruciate ligament reconstruction: predictors of failure from a moon prospective longitudinal cohort. Sports Health 3: 73-81.

13. Pallis M, Svoboda SJ, Cameron KL, et al. (2012) Survival comparison of allograft and autograft anterior cruciate ligament reconstruction at the United States Military Academy. Am J Sports Med 40: 1242-1246.

14. Novak PJ, Wexler GM, Williams, et al. (1996) Comparison of screw post fixation and free bone block interference fixation for anterior cruciate ligament soft tissue grafts: biomechanical considerations. Arthroscopy 12: 470-473.

15. Marder RA, Raskind JR, Carroll M (1991) Prospective evaluation of arthroscopically assisted anterior cruciate ligament reconstruction. Patellar tendon versus semitendinosus and gracilis tendons. Am J Sports Med 19: 478-484.

16. Grawe B, Smerina A, Allen A (2014) Avoiding graft-tunnel length mismatch in anterior cruciate ligament reconstruction: The single-bone plug technique. Arthrosc Tech 3: e417-e420.

17. Auge WK, Yifan K (1999) A technique for resolution of graft-tunnel length mismatch in central third bone patellar tendon bone anterior cruciate ligament reconstruction. Arthroscopy 15: 877881.

18. Verma N, Noerdlingger M, Hallab N, et al. (2003) Effects of graft rotation on initial biomechanical failure characteristics bone bone-patellar tendon-bone constructs. Am J Sports Med 31: 708-713.

19. Shaffer B, Gow W, Tibone JE (1993) Graft-tunnel mismatch in endoscopic anterior cruciate ligament reconstruction: a new tech- nique of intraarticular measurement and modified graft harvesting. Arthroscopy 9: 633-646.

20. Sanders TL, Maradit Kremers H, Bryan AJ, et al. (2016) Incidence of anterior cruciate ligament tears and reconstruction: A 21-year population-based study. Am J Sports Med 44: 1502-1507.

21. Neeraj S (2018) International Epidemiology of Anterior Cruciate Ligament Injuries. Ortho Res Online J 1.

22. Miller D, Hinkin MDT (1996) The "N + 7 rule" for tibial tunnel placement in endoscopic anterior cruciate ligament reconstruction. Arthroscopy 12: 124-126.

23. Joyner P, Wilcox L, Frerichs T, et al. (2013) Quntification of native anterior cruciate ligament length via measurement of lateral radiograph. AANA Annual Meeting, San Antonio TX 4/13.

24. Joyner P, Wilcox L, Frerichs T, et al. (2015) Pre-operative templating of anterior cruciate ligament reconstruction using lateral radiograph to prevent graft tunnel mismatch. 2014 AANA Annual Meeting, Hollywood FL 5/14, AAOS Annual Meeting, Las Vegas NV $3 / 15$

25. Denti M, Bigoni M, Randelli P, et al. (1998) Graft-tunnel mismatch in endoscopic anterior cruciate ligament reconstruction. Intraoperative and cadaver measurement of the intra-articular graft length and the length of the patellar tendon. Knee Surg Sports Traumatol Arthrosc 6: 165-168.

26. Brown JA, Brophy RH, Franco J, et al. (2007) Avoiding allograft length mismatch during anterior cruciate ligament reconstruction: Patient height as an indicator of appropriate graft length. Am J Sports Med 35: 986-989.

27. Verma NN, Dennis MG, Carreira DS, et al. (2005) Preliminary clinical results of two techniques for addressing graft tunnel mismatch in endoscopic anterior cruciate ligament reconstruction. J Knee Surg 18: 183-191.

28. Kenna B, Simon TM, Jackson DW, et al. (1993) Endoscopic ACL reconstruction: $A$ technical note on tunnel length for interference fixation. Arthroscopy 9: 228-230.

29. Olszewski AD, Miller MD, Ritchie JR (1998) Ideal tibial tunnel length for endoscopic anterior cruciate ligament reconstruction. Arthroscopy 14: 9-14.

30. Barber FA (2000) Flipped patellar tendon autograft anterior cruciate ligament reconstruction. Arthroscopy 16: 483-490.

31. Fowler BL, DiStefano VJ (1998) Tibial tunnel bone grafting: A new technique for dealing with graft-tunnel mismatch in endoscopic anterior cruciate ligament reconstruction. Arthroscopy 14: 224228.

32. Madhu S, Saravanakumar KP (2017) Patellar Tendon Graft for Anterior Cruciate Ligament When to Use It. Int J Sci Stud 5: 1-3.

DOI: $10.36959 / 453 / 534$

Copyright: (C) 2020 Eskew JR, et al. This is an open-access article distributed under the terms of the Creative Commons Attribution License, which permits unrestricted use, distribution, and reproduction in any medium, provided the original author and source are credited. 